\title{
Facomatosis pigmentovascular tipo cesioflammea: a propósito de un caso
}

Phakomatosis pigmentovascularis cesioflammea: a case report

\author{
Dra. María C. Viada Peláez ${ }^{a}$ Dra. Paola C. Stefano ${ }^{a}$ Dra. Ana Cirio y Dra. Andrea B. Cervini ${ }^{a}$
}

\begin{abstract}
RESUMEN
La facomatosis pigmentovascular es un síndrome caracterizado por la coexistencia de malformación vascular capilar con una lesión pigmentada cutánea congénita (nevo epidérmico, nevo spilus o melanocitosis dérmica). Existen diversos grupos de facomatosis pigmentovascular que dependen del tipo de nevo asociado a la lesión vascular. Los pacientes pueden presentar solo la afección cutánea o tener manifestaciones sistémicas, entre ellas, traumatológicas, neurológicas y oftalmológicas. Seexponeelcasodeuna niña deunañodeedad, conantecedentes de glaucoma congénito. Además, presentaba parálisis facial, melanosis ocular bilateral, malformación vascular capilar en la hemicara al igual que en el hemicuerpo izquierdo y mancha mongólica aberrante en la región dorsal superior, la zona lumbosacra y ambos glúteos. Debido a las manifestaciones clínicas cutáneas, se diagnosticó facomatosis pigmentovascular y se solicitó una evaluación neurológica y traumatológica, además de continuar con controles oftalmológicos.

Los estudios complementarios son importantes para detectar alteraciones extracutáneas, como se presentaron en esta paciente.

Palabras clave: facomatosis pigmentovascular, malformación vascular capilar, mancha mongólica, facomatosis cesioflammea, niño.
\end{abstract}

\begin{abstract}
Phakomatosis pigmentovascularis (PPV) is a syndrome characterized by the association of a vascular nevus with a congenital pigmented lesion (epidermal nevus, nevus spilus, and dermal melanocytosis). There are different types of PPV according to the pigmentary nevus associated with the vascular malformation. Patients may present only the cutaneous condition or havesystemicmanifestations, among them, trauma, neurological and ophthalmological disorders.

We report the case of a 1-year-old girl who had congenital glaucoma. On examination, we identified facial paralysis, bilateral ocular melanosis, segmental capillary vascular malformation on the face as on left trunk and extremities, and aberrant Mongolian spots on the upper back, lumbosacral area and buttocks. Due to clinical manifestation, the diagnosis of PPV was made. The patient was evaluated by Neurology, Traumatology and keeps on with ophthalmological controls.
\end{abstract}

a. Servicio de Dermatología, Hospital Nacional de Pediatría "Prof. Dr. J. P. Garrahan",

Ciudad Autónoma de Buenos Aires.

Correspondencia:

Dra. María C. Viada Peláez: candeviada@hotmail.com

Financiamiento: Ninguno.

Conflicto de intereses: Ninguno que declarar.

Recibido: 16-5-2017

Aceptado: 13-8-2017
Complementary studies are important, to rule out extracutaneous manifestations in PPV.

Key words: phakomatosis pigmentovascularis, capillary vascular malformation, Mongolian spot, phakomatosis cesioflammea, child.

http: / / dx.doi.org/10.5546/ aap.2018.e121

Cómo citar: Viada Peláez MC, Stefano PC, Cirio A, et al. Facomatosis pigmentovascular tipo cesioflammea: a propósito de un caso. Arch Argent Pediatr 2018;116(1):e121-e124.

\section{INTRODUCCIÓN}

La facomatosis pigmentovascular (FPV) es una enfermedad infrecuente descrita por primera vez en 1947 por Ota y colaboradores como la asociación de un nevo vascular telangiectásico con un nevo pigmentario y verrugoso. ${ }^{1}$ Posteriormente, Hasegawa y Yasuhara agregan a la primera clasificación nuevos subtipos. ${ }^{2}$ Más tarde, Ruiz-Maldonado propone el término de FPV, al encontrar asociación de malformaciones vasculares capilares (MVC) con pigmentación oculocutánea y alteración neurológica grave, y la subdivide según si había compromiso extracutáneo o no, interpretación muy dificultosa. ${ }^{2}$

Finalmente, en 2005, Happle publica una nueva clasificación, que resume las 10 categorías previas con la intención de eliminar la confusión en la nomenclatura y priorizar la descripción clínica de las lesiones. Quedan, así, conformados tres tipos definidos: cesioflammea, spilorosea y facomatosis de tipo cesiomarmorata. ${ }^{3}$ Además de estos grupos bien definidos, existe un cuarto grupo de FPV no clasificable, de los cuales podrán surgir nuevos subtipos de FPV en el futuro, como sucedió con Almeid et al., quienes utilizaron el término melanorosea y otros autores propusieron el subtipo melanovascularis. ${ }^{4}$

Se presenta a una niña de 1 año de edad con manifestaciones clínicas de facomatosis tipo cesioflammea con compromiso cutáneo, oftalmológico y neurológico. 


\section{CASO CLÍNICO}

Paciente de sexo femenino de 1 año de edad, nacida a término, con peso adecuado para la edad gestacional, sin antecedentes familiares patológicos, que presentó glaucoma congénito en el ojo izquierdo y requirió corrección quirúrgica en 2 oportunidades. A los 6 meses de edad, fue derivada a nuestro servicio por la presencia de diferentes máculas cutáneas presentes desde el nacimiento.

Al momento del examen físico dermatológico, la paciente presentaba una mácula eritematosa, extensa, de aspecto reticular que se extendía desde la cabeza hasta el pie izquierdo; se detenía en la línea media corporal y comprometía, de esta forma, la hemicara izquierda y el hemicuerpo homolateral, compatible con MVC.

Además, en la cara, se observaban, en ambas conjuntivas bulbares, máculas grisáceas compatibles con melanosis ocular, con aumento del tamaño del globo ocular izquierdo producto del glaucoma y retracción de la comisura labial de ese mismo lado, debido a la presencia de parálisis facial (Figuras 1 y 2).

FIGURA 1. Mácula eritematosa de aspecto reticular en la hemicara izquierda y el hemicuerpo homolateral (malformación vascular capilar-MVC-). Aumento del tamaño del globo ocular izquierdo. Retracción de la comisura labial



En la región dorsal superior, la paciente presentaba una mácula de coloración azulada grisácea, que se extendía desde la región lumbosacra hacia ambos glúteos, compatible con mancha mongólica aberrante (MMA), y se superponía en algunos sectores con la MVC (Figura 3), por lo que se confirmó el diagnóstico de FPV de tipo cesioflammea. El subtipo cesioflammea surge de la asociación de la mancha mongólica (MM) con la malformación vascular capilar, a pesar de que dicha lesión sea del tipo reticular.

A fin de descartar otros compromisos extracutáneos, se solicitó la evaluación y el seguimiento por los servicios de Clínica Pediátrica, Traumatología, Neurología y Oftalmología. Los estudios complementarios realizados (ecografía abdominal, ecografía cerebral, resonancia magnética nuclear-RMNde cerebro) fueron normales.

En el seguimiento clínico de la paciente, al año de vida, se observó una atenuación de MMA, al igual que de la MVC, y persistió una ligera mácula eritematosa en la región anterior del tórax.

FIGURA 2. Malformación vascular capilar en el hemicuerpo izquierdo y mancha mongólica aberrante en ambos flancos

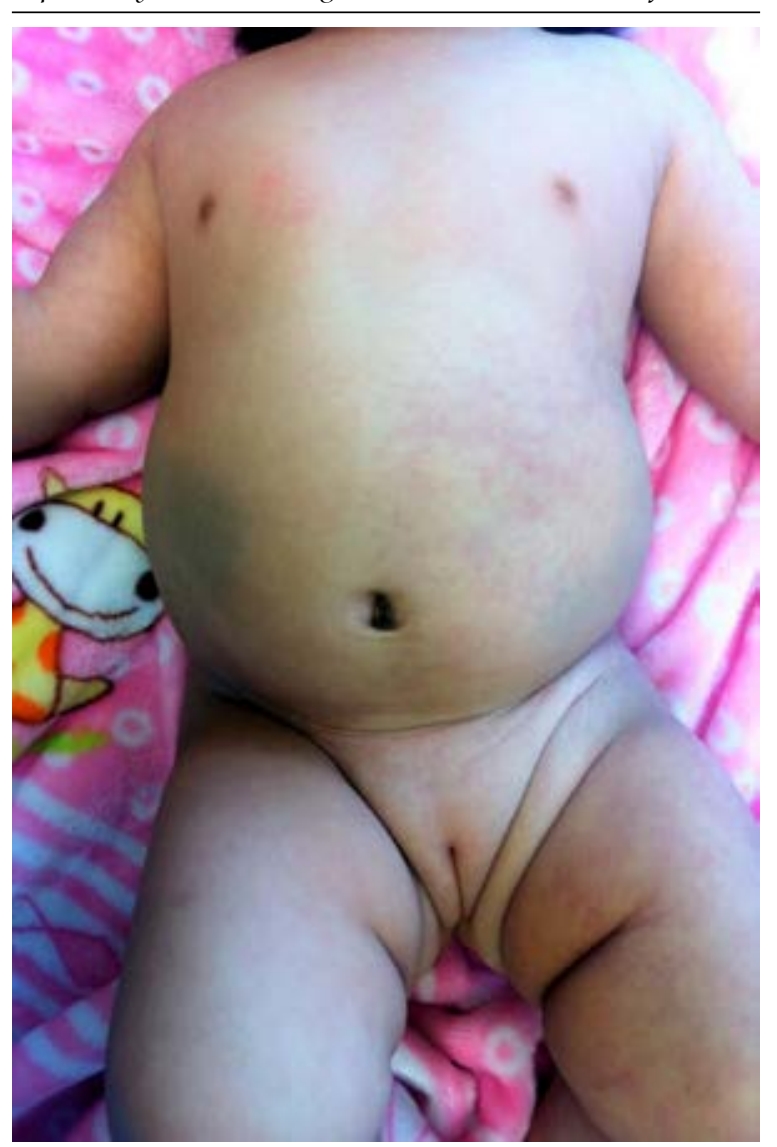




\section{DISCUSIÓN}

La palabra griega phakos significa 'nevus' y la palabra "facomatosis" es empleada

FigURA 3. Malformación vascular capilar en coexistencia con mancha mongólica aberrante en la región dorsal lumbosacra y ambos glúteos

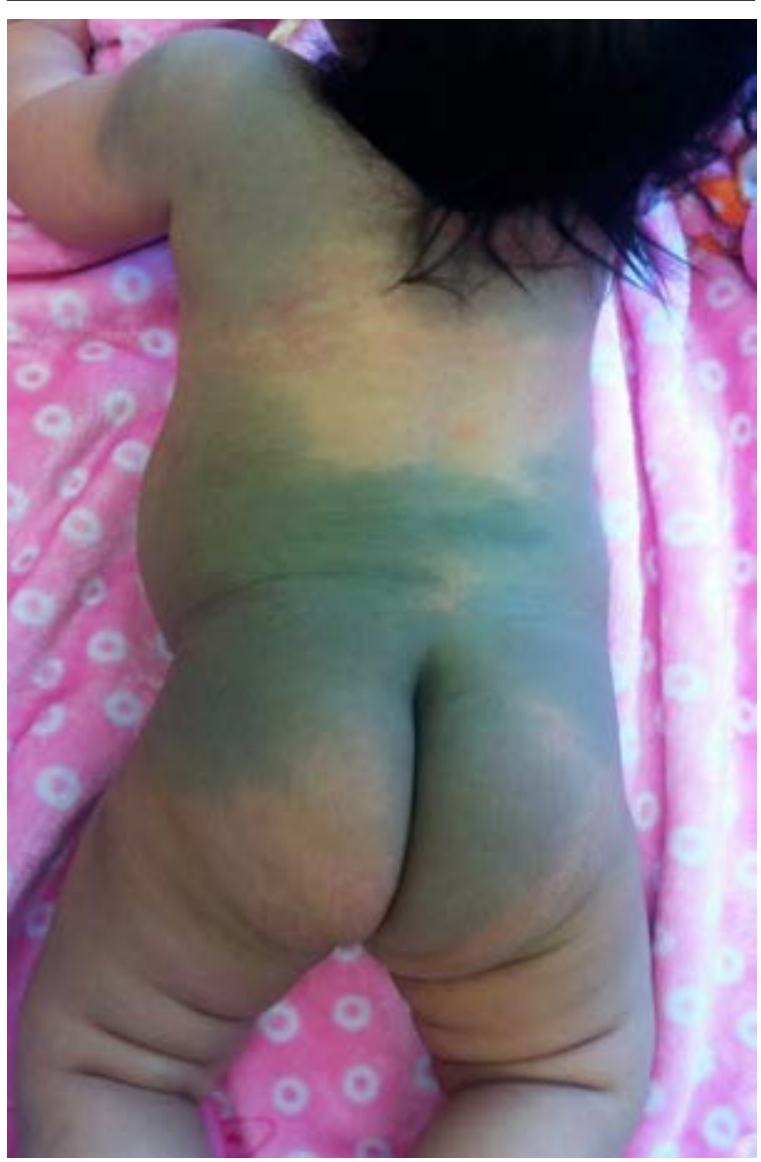

para categorizar patologías que presentan dos o más tipos de nevus, por ejemplo, facomatosis pigmentovascularis o facomatosis pigmentoqueratótica. ${ }^{5}$ Actualmente se propone una nueva clasificación de las facomatosis pigmentovasculares (Tabla 1). ${ }^{6}$

La facomatosis cesioflammea se caracteriza por la coexistencia de nevo flammeus con MM, también llamada nevo cesius; el tipo spilorosea corresponde a la presencia de nevo de spilus con nevo telangiectásico de tipo rosa pálido y la facomatosis de tipo cesiomarmorata se define por la asociación de MM sobre una cutis marmorata telangiectásica congénita. ${ }^{7} \mathrm{El}$ cuarto tipo, facomatosis pigmentovascularis no clasificable, es la combinación de varios tipos de nevus vasculares y pigmentarios no descrita previamente.

La patogenia de la asociación de las extensas anomalías vasculares y pigmentarias en un mismo paciente es desconocida, aunque se ha propuesto para su explicación un modelo genético conocido como twin spots (manchas gemelas) o didimosis. ${ }^{8}$ Este mecanismo da como resultado la presencia, en una misma región del tegumento, de dos áreas de tejido mutante, diferentes entre sí, y del tejido circundante normal. Puede ser causado por azar o bien puede ser explicado por mecanismos biológicos. Durante la división celular, puede producirse una recombinación de algunos fragmentos cromosómicos entre dos cromosomas. Si este fenómeno afecta a una célula heterocigota, puede dar lugar a dos células hijas, que se convertirán en las células madre de dos clones celulares diferentes. ${ }^{9}$ La didimosis sería el mecanismo propuesto para explicar la presencia

Tabla 1. Clasificación de Happle de facomatosis pigmentovascular

\begin{tabular}{lccc}
\hline \multicolumn{2}{c}{ Nueva clasificación propuesta para facomatosis pigmentovascular y su equivalencia con la clasificación previa } \\
\hline Tipo nuevo propuesto & Asociación de nevus & $\begin{array}{c}\text { Equivalencia con la } \\
\text { clasificación previa }\end{array}$ & $\begin{array}{c}\text { Lesiones asociadas } \\
\text { descritas en la literatura }\end{array}$ \\
\hline Facomatosis cesioflammea & Nevus flammeus y nevus cesius & II a, II b & $\begin{array}{l}\text { Nevo anémico, área sin } \\
\text { vello, uñas hipoplásicas }\end{array}$ \\
Facomatosis spilorosea & $\begin{array}{l}\text { Nevus spilus (tipo macular) y nevus } \\
\text { telangiectásico de tipo rosado pálido }\end{array}$ & III a, III b & $\begin{array}{l}\text { Áreas de alopecia, tumores } \\
\text { de células granulares, } \\
\text { linfedema }\end{array}$ \\
Facomatosis cesiomarmorata & $\begin{array}{l}\text { Nevus cesius y cutis marmorata } \\
\text { telangiectásica congénita }\end{array}$ & V a, V b & $\begin{array}{l}\text { Manchas café con leche, } \\
\text { máculas hipomelanóticas, } \\
\text { nevo sebáceo }\end{array}$ \\
FPV no clasificable & $\begin{array}{l}\text { Varios tipos de nevus vasculares y } \\
\text { pigmentarios asociados a facomatosis } \\
\text { cesioflammea o facomatosis spilorosea }\end{array}$ & IV a, IV b, otros & \\
\hline
\end{tabular}

FPV: facomatosis pigmentovascular. 
de varios tipos de lesiones cutáneas en la FPV..$^{10}$ Sin embargo, una observación interesante ${ }^{11}$ se realizó en pacientes con diagnóstico de facomatosis pigmentoqueratótica (asociación de nevo lentiginoso moteado de tipo papular y nevo epidérmico organoide), en los que la patogenia estaba sostenida por el mecanismo de didimosis. Los autores mostraron una activación poscigótica del gen HRAS en células progenitoras pluripotentes que dieron lugar a nevus melanocíticos y nevus sebáceo, y, por lo tanto, sostuvieron la hipótesis de rasopatía. Transpolando esta hipótesis a otras patologías en las que se fundamenta la didimosis, Nanda et al., en su publicación de cuatro casos clínicos de $\mathrm{FPV},{ }^{12}$ negaron la hipótesis de manchas gemelas (twin spots).

Asimismo, recientes investigaciones ${ }^{13}$ identificaron mutaciones en el gen GNAQ [proteína de unión a nucleótidos de guanina (proteína G)] en pacientes con manchas de vino de Oporto en el síndrome de Sturge-Weber, nevos azules, melanoma uveal y nevo de Ota. Todas estas manifestaciones cutáneas han sido reportadas en asociación con FPV.

Nuestra paciente presentaba un cuadro clínico compatible con FPV, teniendo en cuenta la clasificación de Happle (Tabla 1), de tipo cesioflammea con compromiso sistémico debido a la presencia de glaucoma congénito y parálisis facial.

Es importante realizar el diagnóstico de FPV en el período neonatal para poder evaluar en forma precoz el compromiso extracutáneo. ${ }^{13}$ Debe realizarse una evaluación multidisciplinaria para descartar la presencia de asimetría de los miembros, malformaciones neurológicas (calcificaciones intracraneales, angiomatosis leptomeníngea o atrofia cerebral) y compromiso ocular (glaucoma, melanosis ocular con posibilidad de desarrollar melanoma), entre las asociaciones extracutáneas más frecuentes. ${ }^{14}$

La evolución y el pronóstico de la FPV dependen de las manifestaciones extracutáneas, ya que los pacientes sin compromiso sistémico tienen un curso benigno y no requieren tratamiento. Teniendo en cuenta el impacto estético que pueden ocasionar las lesiones cutáneas, existe la posibilidad de realizar como terapéutica láser colorante pulsado para MVC y láser $\mathrm{Q}$ switched para los nevos pigmentarios. ${ }^{15}$

\section{CONCLUSIÓN}

La FPV es una patología infrecuente en la práctica pediátrica, que debe sospecharse ante la presencia de MVC y MMA. Se deben tener presentes las posibles asociaciones con trastornos neurológicos, oftalmológicos y óseos.

\section{REFERENCIAS}

1. Ota M, Kawamura $\mathrm{T}$, Ito $\mathrm{N}$. Phakomatosis pigmentovascularis. Jpn J Dermatol 1947;52:1-3.

2. QuirozC,SilvaS.FacomatosisPigmentovascularis:Revisión y Nueva Clasificación. Rev Chil Dermatol 2010;26(1):36-40.

3. Fernández-Guarino M, Boixeda P, De Las Heras E, et al. Phakomatosis pigmentovascularis: Clinical findings in 15 patients and review of the literature. J Am Acad Dermatol 2008;58(1):88-93.

4. Arnold AW, Kleine MU, Happle R. Phacomatosis melanorosea without extracutaneous features: an unusual type of phacomatosis pigmentovascularis. Eur J Dermatol 2012;22(4):473-5.

5. Happle R. Neurocutaneous diseases. In: Freedberg IM, Eisen AZ, Wolff K, et al eds. Fitzpatrick's Dermatology in General Medicine. 6th ed. New York: Mc Graw-Hill; 2003. Págs.1806-21.

6. Happle R, Phakomatosis pigmentovascularis revisted and reclassified. Arch Dermatol 2005;141:385-8.

7. HasegawaY,YasuharaM.Phakomatosispigmentovascularis type IVa. Arch Dermatol 1985;121(5):651-5.

8. Happle R. Mosaicism in human skin, understanding the patterns and mechanisms. Arch Dermatol 1993;129(11): 1460-70.

9. Happle R. New aspects of cutaneous mosaicism. J Dermatol 2002;29(11):681-92.

10. Rodríguez-Díaz E, Álvarez Cuesta C, Blanco S, et al. Nevo de Becker asociado a nevo epidérmico: ¿un ejemplo más de «manchas gemelas»? Actas Dermosifiliogr 2006;97(3):200-2.

11. Groesser L, Herschberger E, Sagrera A, et al. Phacomatosis pigmentokeratotica is caused by a postzygotic HRAS mutation in a multipotent progenitor cell. J Invest Dermatol 2013;133(8):1998-2003.

12. Nanda A, Al-Abdulrazzaq HK, Habeeb $Y K$, et al. Phacomatosis pigmentovascularis: Report of four new cases. Indian J Dermatol Venereol Leprol 2016;82(3):298-303.

13. Thomas A, Zeng Z, Rivière JB, et al. Mosaic Activating Mutations in GNA11 and GNAQ are associated with phakomatosis pigmentovascularis and extensive dermal melanocytosis. J Invest Dermatol 2016;136(4):770-8.

14. Vidaurri-de la Cruz H, Tamayo-Sánchez L, Durán McKinster $\mathrm{C}$, et al. Phakomatosis pigmentovascularis II A and II B: Clinical findings in 24 patients. J Dermatol 2003;30(5):381-8.

15. Segatto MM, Schmitt E, Hagemann L, et al. Phacomatosis pigmentovascularis type IIa - Case report. An Bras Dermatol 2013;88(6 Suppl 1):S85-8. 